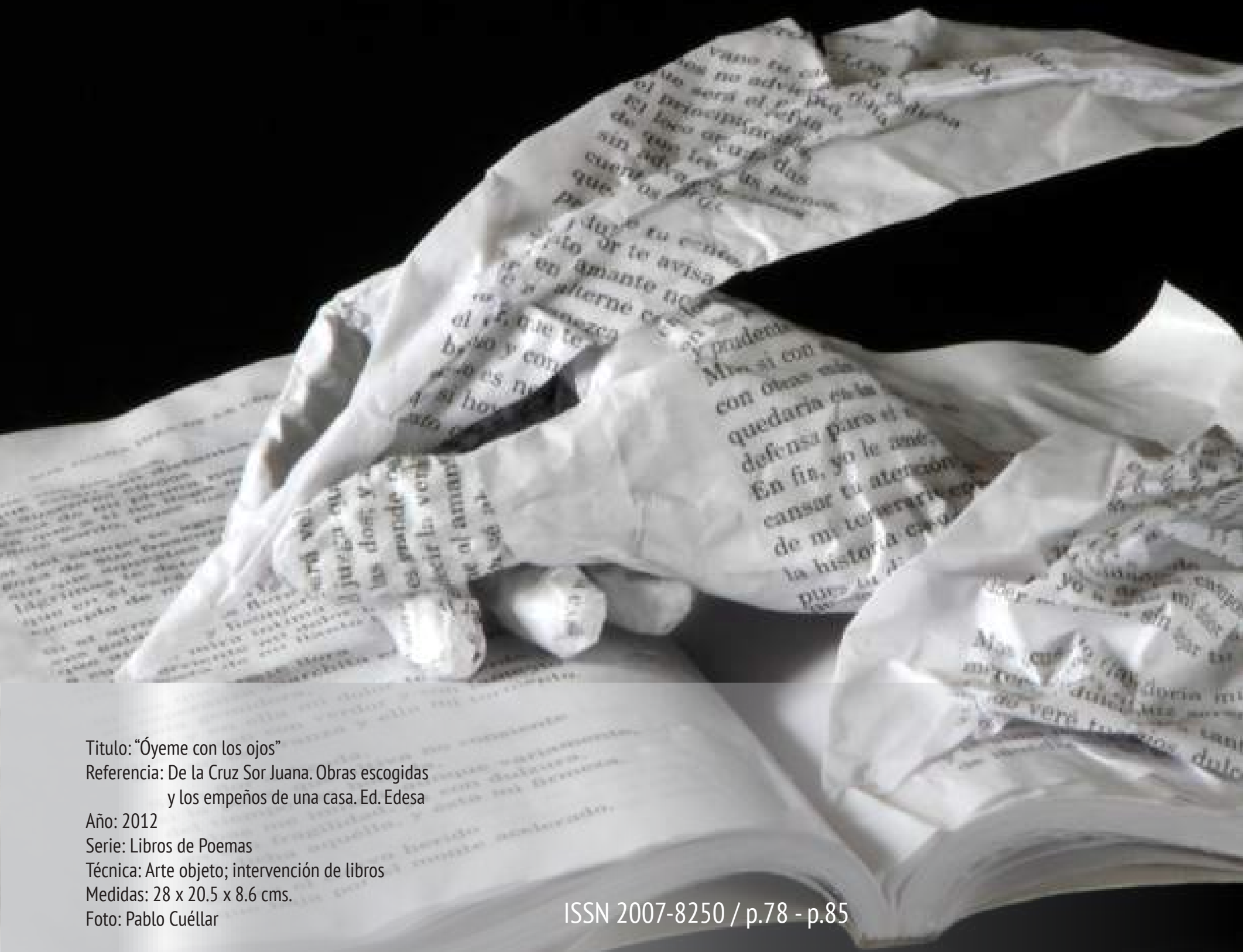




\title{
Factores relevantes en la planificación de los procesos de enseñanza aprendizaje y la práctica docente por competencias en el nivel medio superior
}

\author{
Relevant factors in the planning of the teaching-learning processes \\ and the teaching practice by competences in the high school level
}

Esperanza Escobedo Castañeda

\section{Resumen}

La presente investigación se basó en el estudio de la competencia docente que trata sobre la planificación del proceso de enseñanza aprendizaje y sus respectivas propiedades atendiendo el enfoque por competencias, en los docentes cuyos contextos disciplinares son las unidades de aprendizaje de los bachilleres técnicos del área médica en la Escuela y Preparatoria Técnica Médica de la Universidad Autónoma de Nuevo León. Con la finalidad de determinar la operatividad de la competencia docente referida, se revisó su relación con la aplicación de las estrategias didácticas que conduzcan al logro óptimo del aprendizaje significativo; así como la planeación de proyectos disciplinares y el uso de materiales apropiados para el desarrollo de competencias en su contextualización y su realidad social.

Palabras clave: planificación, enseñanza, aprendizaje, competencias, médica, docente.

\begin{abstract}
The present investigation was based on the study of the teaching competence that deals with the planning of the teachinglearning process and its respective properties attending the competency-based approach, in the teachers whose disciplinary contexts are the learning units of the technical baccalaureates of the medical area at the Escuela y Preparatoria Técnica Médica of the Universidad Autónoma de Nuevo León.. In order to determine the operability of the aforementioned teaching competence, its relationship with the application of the didactic strategies that lead to the optimal achievement of meaningful learning was reviewed; as well as the planning of disciplinary projects and the use of appropriate materials for the development of competencies in their contextualization and their social reality
\end{abstract}

Keywords: planning, teaching, learning, competencies, medical, teacher. 
a presente investigación correspondió al tipo de estudio cuantitativo a través del análisis de datos y la descripción e interpretación de resultados. Se empleó una encuesta estructurada de setenta preguntas cuyos contenidos tratan sobre las variables a ser estudiadas. Se resalta la participación de los docentes en su operatividad didáctica en cada una de las actividades educativas desde la planeación, hasta su aterrizaje en diversos contextos $\mathrm{Cu}$ rriculares.

Pertenece al tipo no correlacional ya que no se está midiendo la relación entre dos o más variables, por lo que se ha centrado en el análisis y descripción en forma cuantitativa en cada una de ellas. De esta manera se puede concluir que los docentes involucrados en el estudio de la investigación, están llevando a la práctica en un alto porcentaje las competencias planeadas.

Las competencias en la educación se definen como el conjunto de conocimientos, habilidades y destrezas, tanto específicas como transversales, que debe reunir un titulado para satisfacer plenamente las exigencias sociales y fomentar las competencias como objetivo de los programas educativos. Las competencias son capacidades que la persona desarrolla en forma gradual y a lo largo de todo el proceso educativo y son evaluadas en diferentes etapas (ANUIES, 2006).

\section{Acuerdo Oficial número 444}

El Marco Curricular Común expresa del Sistema Nacional de Bachillerato que está orientado a dotar a los estudiantes de la Educación Media Superior de una identidad que responda a sus necesidades presentes y futuras. Entre las competencias a que se refiere el presente acuerdo que son las genéricas, las disciplinares y las profesionales.

Se concluyó que las competencias genéricas son las que todos los bachilleres deben estar en capacidad de desempeñar, las que les permiten comprender el mundo e influir en él, que les capacitan para continuar aprendiendo de forma autónoma a lo largo de sus vidas y para desarrollar relaciones armónicas con quienes les rodean, así como participar eficazmente en los ámbitos social, profesional y político.

\section{Competencias Docentes}

Perrenoud (2005), refiere que las competencias docentes son las que representan una capacidad de movilizar varios recursos cognitivos para hacer frente a un tipo de situaciones. De acuerdo con Perrenoud, la descripción de una competencia implica tres elementos: las situaciones; los recursos que moviliza; la naturaleza de los esquemas de pensamiento que permiten la movilización y la orquestación de los recursos pertinentes en situaciones complejas y en tiempo real.

\section{Acuerdo Oficial 447}

En este acuerdo se establecen y se describen ocho competencias docentes con sus respectivos cuarenta atributos, para ser empleadas como el enfoque integral en los contextos educativos afines en que sea necesaria su aplicación para el logro de los aprendizajes esperados en el nivel medio superior. Estudiar la educación por competencias en cualquiera de los niveles de enseñanza,

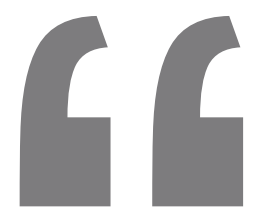

Las competencias

en la educación

se definen como

el conjunto de

conocimientos,

habilidades y

destrezas, que

debe reunir un

titulado para

satisfacer las

exigencias sociales

y fomentar las

competencias". 
nos induce a referir los antecedentes encontrados que sobre el concepto de competencia se ha encontrado en el ámbito educativo. Resaltando que el tema de competencias, empezó a ser empleado en el campo educativo a finales de la década de los cincuenta del siglo pasado, por la Gramática Generativa de Chomsky, quien emplea el término de competencias al referirse a las capacidades y conocimientos que debe tener un hablante oyente.

En los años setenta países como Canadá, Reino Unido, Australia, Alemania, España, México, Colombia, Perú y Jamaica, adoptaron la Educación Basada en Competencias (EBC) y no sólo fue en el campo de las habilidades. Sin embargo, fue hasta los años ochenta cuando el concepto de competencias es redefinido en los marcos del debate internacional en torno a los problemas de la educación de calidad.

La educación basada en competencias surgió en ambos lados de la frontera entre los Estados Unidos de América y Canadá durante la década de los setenta como respuesta a la crisis económica, cuyos efectos en la educación afectaron sensiblemente a todos los países. Al mismo tiempo el mundo enfrentó, en esa década, un incremento considerable en la demanda de educación media superior, ocasionado por la dinámica de la población ya que la mayoría de ellos tenía entre quince y veinticinco años de edad.

Desde hace aproximadamente dos décadas el Colegio Nacional de Educación Profesional Técnica (CONALEP) trajo a México la educación y la capacitación basada en competencias, en los estudios ofrecidos a jóvenes en el nivel medio superior.

En esta propuesta de investigación lo que se pretende es determinar la operatividad de la competencia docente en relación a la planificación del proceso de enseñanza-aprendizaje y con base al enfoque por competencias, aunada al desarrollo de las estrategias didácticas que, al llevarlas a cabo en la educación media superior del área médica, nos conduzcan al logro óptimo del aprendizaje asociado a la práctica y desarrollo de las competencias de los estudiantes.

Además de identificar lo que los docentes planean a través de proyectos e investigaciones tanto disciplinarios como interdisciplinarios y de actividades para su realización en el aula considerando la acertada contextualización de los saberes de los estudiantes.

\section{Objetivos de la investigación}

1. Identificar la participación del docente en la planificación del proceso de enseñanza aprendizaje en las unidades de aprendizaje de los bachilleratos técnicos del área médica.

2. Valorar la identificación de los conocimientos previos y la selección y desarrollo de las estrategias didácticas que los docentes de los bachilleres técnicos del área médica utilizan en la formación integral de los estudiantes.

3. Identificar la planificación que los docentes realizan a través de planes de trabajo basados en proyectos e investigaciones disciplinarias e interdisciplinarias orientados al desa-

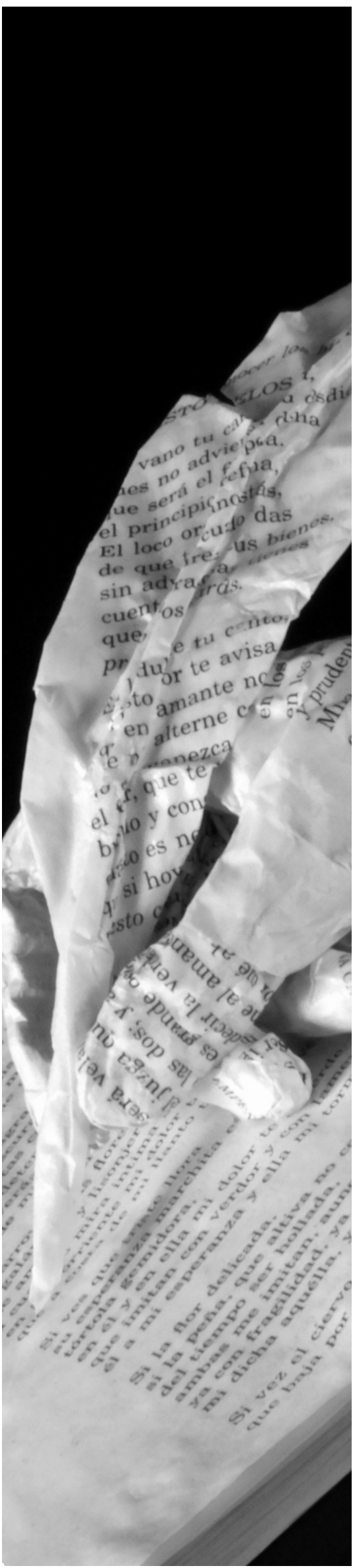

Presencia Universitaria 
rrollo de competencias académicas en las unidades de aprendizaje de los bachilleratos técnicos del área médica.

4. Valorar el diseño y elaboración de materiales y recursos didácticos que realiza el docente como herramienta para el desarrollo de competencias, en el salón de clase con los estudiantes de los bachilleratos técnicos del área médica.

5. Identificar la tarea de los docentes en la contextualización de contenidos y actividades de aprendizaje relativas al plan de estudios de los estudiantes y la realidad social de la comunidad a la que pertenecen.

La hipótesis que se plantea es la siguiente: Los docentes de los bachilleratos técnicos del área médica realizan la planificación de los procesos de enseñanza aprendizaje atendiendo el enfoque por competencias en contextos disciplinares, curriculares y sociales amplios.

La razón principal por la que se hace esta investigación es indagar la operatividad didáctica realizada por los docentes de las unidades de aprendizaje del área técnica médica, considerando los saberes previos, los conocimientos esperados, el uso acertado de estrategias, el considerar los materiales didácticos suficientes, además de su labor realizada con los estudiantes al estar llevando a cabo las actividades suficientes en el ejercicio de sus competencias.

Determinar el desarrollo y la aplicación de la competencia que están trabajando los docentes en el salón de clase, son en conjunto, una serie de actividades que el modelo de educación por competencias demanda, ya que son los estudiantes de los bachilleratos técnicos del área médica los mayormente beneficiados en cuanto a las actividades docentes acordes al desarrollo óptimo de las competencias.

El docente siempre ha llevado a cabo el uso de competencias sin seguir un proceso, por lo que es significativo ordenar y proponer un cambio en el rubro de éstas y, si éste fuera necesario, que de ahí parta el docente para la realización de clase. Una buena propuesta en la aplicación y desarrollo de las competencias docentes sobre la planificación de los procesos de enseñanza aprendizaje en el ámbito educativo de los bachilleres técnicos del área médica, repercutirá en la calidad y resultados efectivos en la clase áulica y será punta de partida en posteriores investigaciones que sobre el tema sean realizadas.

La Secretaría de Educación del país planteó la conformación de un Sistema Nacional de Bachillerato bus- cando fortalecer la identidad de la educación media superior, donde se toma como eje el enfoque de competencias para la estructuración de un Marco Curricular Común. En lo que respecta a la práctica diaria de los docentes del nivel bachillerato, estos deben tener en cuenta en sus planeaciones didácticas el ejercicio de algunas de las ocho competencias docentes que según señala el acuerdo 447 de la Reforma Integral de la Educación Media Superior (RIEMS).

El docente debe poner en práctica sus saberes y movilizar los de los estudiantes a su cargo, reconociendo que no todos son iguales y por lo tanto su aprendizaje será distinto, por lo que habrá un grado de dificultad diferente en cada uno. Deberá diseñar y aplicar diversas estrategias didácticas y métodos acordes a las necesidades, para así lograr la aplicación y desarrollo de competencias tanto docentes como de los estudiantes.

Las competencias deben ser consideradas en forma integral, es decir, se

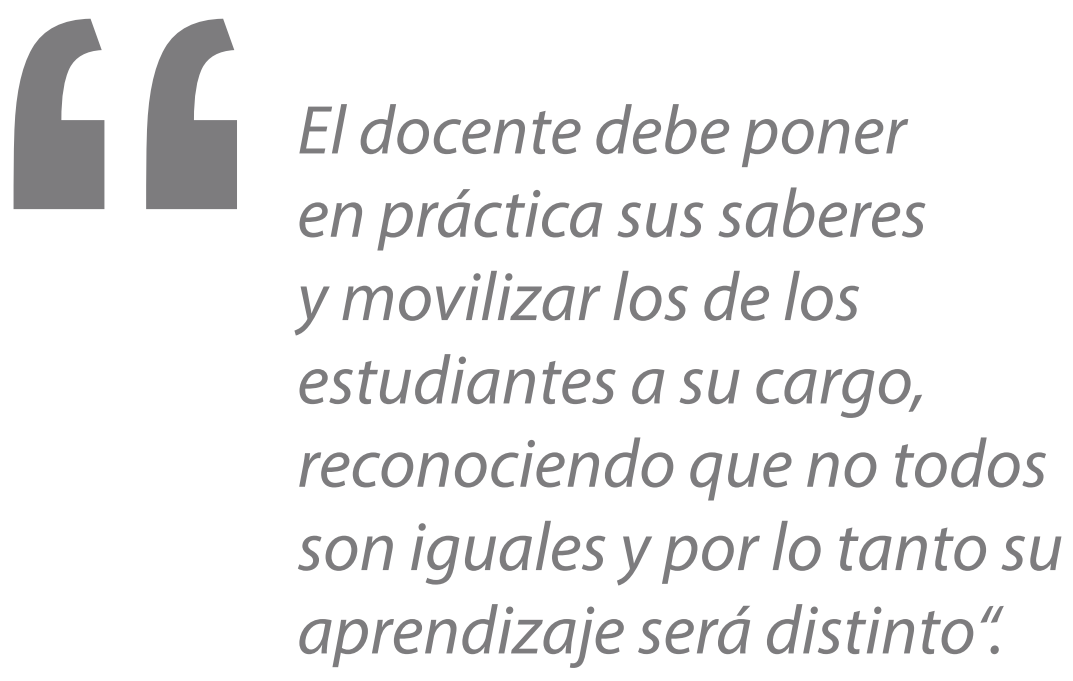


debe centrar la atención en el proceso de construcción de los aprendizajes que en la enseñanza; y es aquí donde el docente se convierte en un facilitador de este proceso, debiendo reestructurar los programas educativos para que el desarrollo de competencias pueda ser abordado de manera integral.

En la práctica, el docente debe crear espacios curriculares y cocurriculares que permitan el desarrollo de competencias en situaciones de aprendizaje reales, así mismo debe establecer esquemas de vinculación con el sistema de investigación, innovación y desarrollo tecnológico de la UANL.

En su implementación los programas de formación docente deben desarrollar las competencias requeridas para su práctica educativa. Se resalta que la universidad, con el fin de certificar la práctica docente con base a competencias, lleva a cabo su certificación través de organismos reconocidos asegurando la educación integral por competencias y facilitando su incorporación a otros contextos (Acuerdos Oficiales de la Federación 442 y 447).

El método para la selección de la muestra en esta investigación es el no probabilístico intencional, eligiéndose un promedio de 45 docentes que imparten clase en alguna de las unidades de aprendizaje del área de los bachilleratos técnicos de la Escuela y Preparatoria Técnica Médica, de los que solamente se obtuvieron respuestas completas en las encuestas de 35 de ellos, siendo éste el

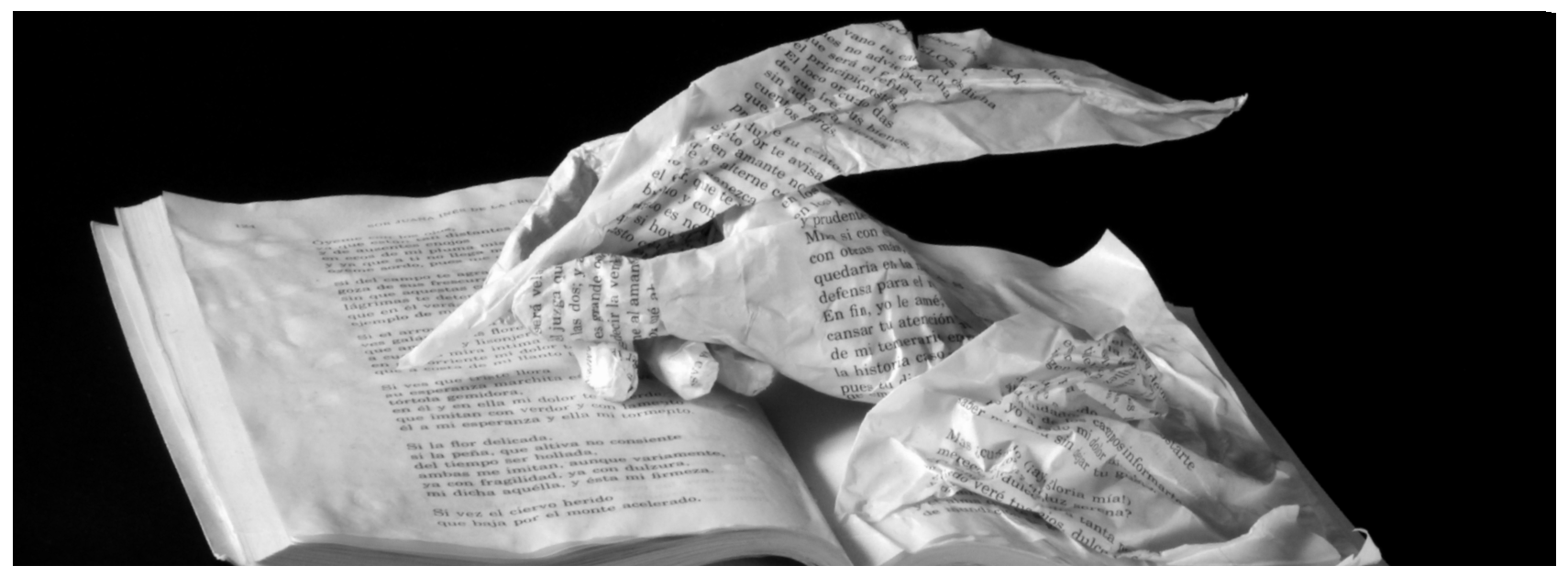

número final que se consideró en el presente trabajo de investigación.

Para la recopilación de la información se elaboró una encuesta de 70 ítems alusivos al tema de investigación, adecuados de acuerdo a cada una de las variables de estudio con la elección de cinco categorías de respuesta que fueron las siguientes:

(1) Totalmente en desacuerdo

(2) En desacuerdo

(3) Ni de acuerdo ni en desacuerdo

(4) De acuerdo

(5) Totalmente de acuerdo
Además se diseñaron 11 ítems alusivos a la identificación de los docentes participantes en forma general, como son la formación y capacitación académica de los 35 docentes encuestados. Todo el material se procesó con la herramienta del SPSS la cual es un poderoso material en el proceso de datos y análisis estadístico, permitiendo también atender el estudio de los resultados a través de cada uno de los apartados de la encuesta.

El análisis se inició con una fuente de información alineada en una matriz de datos conformada por filas y columnas del programa SPSS, donde el número de ellas fue de acuerdo a cada una de las cinco variables a estudiar. De acuerdo a los resultados de cada una de las variables en el presente estudio de investigación, cuyo propósito ha sido el obtener la información sobre la práctica de la competencia docente que versa sobre la planificación de los procesos de enseñanza aprendizaje en el salón de clase de los bachilleratos técnicos del área médica, se puede deducir que una gran fortaleza es el que se esté llevando a cabo la planificación didáctica al inicio de cada ciclo es- 
colar; sin embargo, se debe poner atención a los ítems con porcentajes altos comparados con los demás en respuesta de totalmente en desacuerdo, ya que se pueden considerar como áreas de oportunidad en el ámbito docente.

Respecto a la información que nos da un perfil alineado a la elección de dos 0 más estrategias en clase y no solamente una de ellas, es altamente significativo el número de docentes que lo está realizando. Se hace relevante el poner atención a la operatividad de la práctica de la investigación con base a proyectos de parte de los docentes.

Se resalta también el atender que solo algunos docentes planean los recursos y materiales a trabajar con base al desarrollo de competencias. Finalizando en resultados que nos señalan el no estar teniendo en cuenta todos los contextos de los estudiantes en el plan de aprendizaje.

En el proceso de enseñanza-aprendizaje estamos expresando que éste es un proceso de retroalimentación continua, sobresaliendo que aunque son competencias denominadas diferentes, en su operatividad didáctica no lo son ya que deben relacionarse en cada una de las actividades educativas desde la planeación hasta su aterrizaje en los diversos contextos curriculares (Tobón, 2008).

En nuestro contexto que es la UANL, se debe considerar que la formación integral del estudiante comprende, además de los aspectos disciplinarios, elementos culturales, deportivos, recreativos, de salud y de desarrollo personal, entre otros, $y$ en general, un mejor desempeño académico (PDI-UANL 2007-2012, 2008).

A continuación se resalta sólo una interrogante que conviene responder a través de posteriores investigaciones: ¿Los docentes conocen en forma integral hacia donde se dirige el proceso de enseñanza-aprendizaje basado en competencias que están poniendo en práctica?

\section{Referencias}

Comisión Europea. (2009). El Marco Europeo de Cualificaciones para el aprendizaje permanente. Bélgica: Oficina de Publicaciones Oficiales de las Comunidades Europeas.

García Cabrero, B. (s.f.) La Educación Basada en Competencias: Aplicaciones al diseño curricular y la evaluación de la docencia. Recuperado de www.iberopuebla.mx/micrositios/ ceamope/docs/1foro/educacioncompetencias.ppt

Perrenoud, P. (2005). La formación de los docentes en el siglo XXI. Revista de Tecnología Educativa, 3(XIV), 503-523. Recuperado de http://academicos.iems.edu.mx/cired/docs/tg/ macroacademiaquimica/La\%20formacion\%20de\%20los\%20docentes\%20en\%20el\%20 siglo\%20XXI_Perrenoud.pdf

SEP.(21 de octubre de 2008). ACUERDO número 444 por el que se establecen las competencias que constituyen el marco curricular común del Sistema Nacional de Bachillerato. Diario Oficial. 1ra Sección, México. Recuperado de http://www.sems.gob.mx/work/models/sems/ Resource/11435/1/images/5_2_acuerdo_444_competencias_mcc_snb.pdf

SEP.(29 de octubre de 2008). ACUERDO número 447 por el que se establecen las competencias docentes para quienes impartan educación media superior en la modalidad escolarizada. Diario Oficial. 3ra Sección, México. Recuperado de http://www.sems.gob.mx/work/models/ sems/Resource/11435/1/images/5_4_acuerdo_447_competencias_docentes_ems.pdf

Tobón, S. (2008). La formación basada en competencias en la Educación Superior: el enfoque complejo. Guadalajara: IGLU.

UANL. (2012). Plan de Desarrollo Institucional UANL 2012-2020. Aprobado por el H. Consejo Universitario el 29 de Marzo de 2012. San Nicolas de los Garza: UANL. Recuperado de http:// www.uanl.mx/sites/default/files/documentos/universidad/pdi-2020-26abril.pdf 


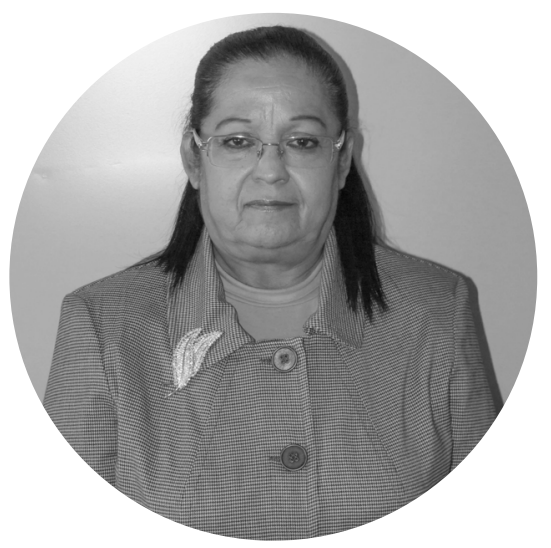

\section{Esperanza \\ Escobedo Castañeda}

Es maestra de tiempo completo de la Universidad Autónoma de Nuevo León, con fecha de ingreso en el año de 1979 en la Escuela y Preparatoria Técnica Médica impartiendo clases en el bachillerato técnico en Enfermería y desde el año 1995 colaborando en la Dirección del Sistema de Estudios del Nivel Medio Superior iniciando en la Coordinación de Bachilleratos Técnicos y actualmente como responsable de la Unidad de Aprendizaje de Introducción a las Actividades Empresariales. Experiencia laboral como docente en la impartición de clase en los niveles medio superior, licenciatura y maestría. Instructora de los módulos I, II y III del programa de PROFORDEMS en todas las generaciones y evaluadora de docentes en el programa de CERTIDEMS.

Correo electrónico:

escobed07@hotmail.com

Recibido: 30/08/2017

Aceptado: 20/11/2017 